Claremont Colleges

Scholarship@ Claremont

All HMC Faculty Publications and Research

HMC Faculty Scholarship

$1-1-2002$

\title{
Theoretical Investigation of the Ground and Excited States of Coumarin 151 and Coumarin 120
}

Robert J. Cave

Harvey Mudd College

Kieron Burke

Rutgers University - New Brunswick/Piscataway

Edward W. Castner Jr.

Rutgers University

\section{Recommended Citation}

Cave, R.J.; Burke, K.; Castner, E. W. Jr. "Theoretical Investigation of the Ground and Excited States of Coumarin 151 and Coumarin 120,” J. Phys. Chem. A 2002, 106, 9294. DOI: 10.1021/jp026071x

This Article is brought to you for free and open access by the HMC Faculty Scholarship at Scholarship @ Claremont. It has been accepted for inclusion in All HMC Faculty Publications and Research by an authorized administrator of Scholarship @ Claremont. For more information, please contact scholarship@cuc.claremont.edu. 


\title{
Theoretical Investigation of the Ground and Excited States of Coumarin 151 and Coumarin 120
}

\author{
Robert J. Cave* \\ Department of Chemistry, Harvey Mudd College, Claremont, California 91711
}

\author{
Kieron Burke ${ }^{\dagger}$ and Edward W. Castner, Jr. ${ }^{\$}$ \\ Department of Chemistry and Chemical Biology, Rutgers University, 610 Taylor Road, \\ Piscataway, New Jersey 08854-8087
}

Received: May 6, 2002

\begin{abstract}
We present calculations of various properties of the ground and excited states of Coumarins 151 and 120 . These and related coumarins are important in investigating ultrafast solvation processes in liquids and complex solutions as well as being important acceptors in model electron-transfer systems. We calculate the following: (1) the electronic excitation energies to several low-lying singlet states, (2) ground and excitedstate dipole moments, (3) solvation effects on excitation energies, and (4) the properties of single Coumarin 151-water complexes. We test our Time-Dependent Density Functional Theory (TDDFT) calculations against CASSCF, CASPT2 (both single and multistate versions), CIS, and ZINDO. Using TDDFT, we find excellent agreement with experimental $\mathrm{S}_{1} \leftarrow \mathrm{S}_{0}$ excitation energies. On the basis of these results, we address several outstanding questions for these systems and find: (1) that TICT-formation is unlikely upon photexcitation for gas-phase $\mathrm{C} 151$, (2) a greater tendency toward a planar amine group for the $S_{1}$ state than for the ground state, (3) significant differences between our gas-phase ground-state dipole moment and the experimental value, and (4) TDDFT results for water-Coumarin 151 complexes are in good agreement with the experimental results of Topp and co-workers.
\end{abstract}

\section{Introduction}

The strong absorption cross sections and large radiative yields of coumarins make them excellent laser dyes at near-ultraviolet to green wavelengths. ${ }^{1}$ The relative rigidity and solvatochromic properties of the coumarins led to wide use as fluorescence probes of condensed phase environments, especially for timedependent fluorescence Stokes shift studies of solvent reorganization $^{2}$ and solute-solvent friction via fluorescence depolarization. ${ }^{3}$ The energy of the lowest singlet state relative to the ground state for 7-aminocoumarins is remarkably sensitive to the polarity of their environment, indicating a substantial dipole moment change between the ground and excited state. ${ }^{4-8}$ This solvatochromic behavior has allowed a number of groups to use ultrafast spectroscopic techniques to unravel the dynamics of solvation in polar solvents by examining coumarin fluorescence dynamics. ${ }^{2,9-16}$ Following this seminal work, researchers have begun to apply coumarin fluorescence dynamics to probe local environment fluctuations in a wide variety of chemical environments. ${ }^{17-29}$ Although the energetics and dynamics of solvation for many coumarins have been well-characterized experimentally, there are still many open questions concerning the nature of the $S_{1}$ state, as well as its evolution from the Franck-Condon region following photoexcitation from the ground state. (See Table 1 for a collection of previous experimental and theoretical results on $\mathrm{C} 151$ and $\mathrm{C} 120$.)

Coumarins can also serve as partners in ultrafast electron transfer (et) reactions in solution. ${ }^{30,31}$ The Yoshihara group has

\footnotetext{
* To whom correspondence should be addressed. E-mail: Robert_Cave@ hmc.edu.

†-mail: kieron@rutchem.rutgers.edu.

E-mail: castner@rutchem.rutgers.edu.
}

TABLE 1: Previous Results for C151

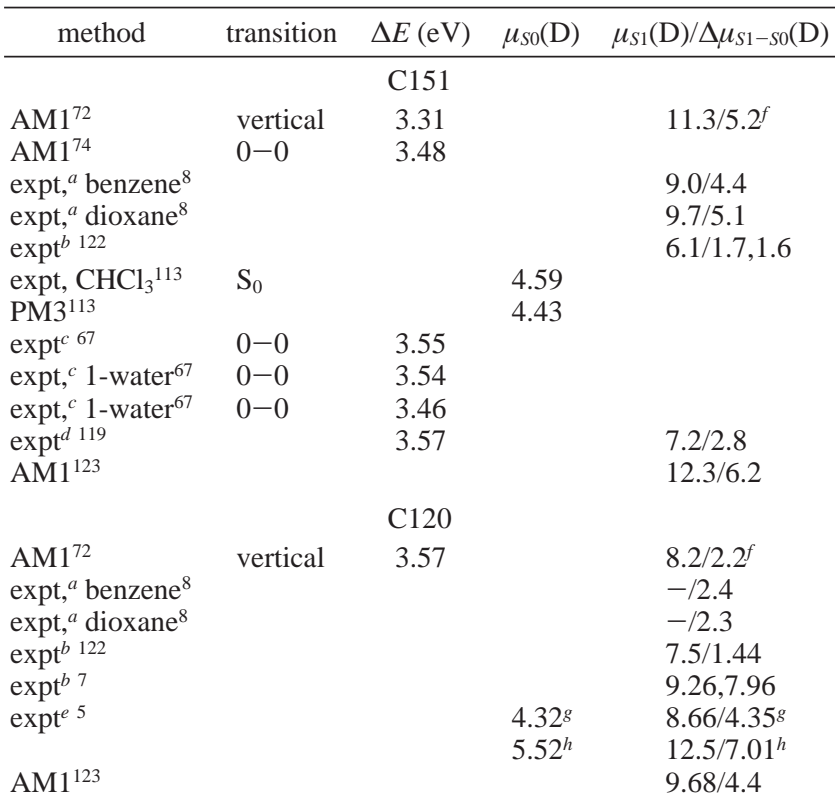

${ }^{a}$ Conductivity. ${ }^{b}$ Solvatochromism. ${ }^{c}$ Gas-phase optical spectroscopy. ${ }^{d}$ Solution-phase optical spectroscopy. ${ }^{e}$ Electroabsorption spectroscopy. ${ }^{f}$ Scalar difference. ${ }^{g}$ Cyclohexane solvent. ${ }^{h}$ Dioxane solvent.

studied solvent to solute et, ${ }^{32-43}$ using coumarins or related species as electron acceptors - the coumarin is photoexcited, and the hole in the HOMO is then a good oxidant for many solvents. Here too, important questions remain open regarding the nuclear coordinate reorganization in the $\mathrm{S}_{1}$ and other excited states, with 


\section{SCHEME 1}<smiles>Nc1ccc2c(C(F)(F)F)cc(=O)oc2c1</smiles>

C151<smiles>Cc1cc(=O)oc2cc(N)ccc12</smiles>

C120 implications for the relative rates of electron transfer, as well as the energetic proximity of other low-lying states.

We have recently examined et between a variety of coumarins and solvents using experimental and theoretical methods. ${ }^{44}$ In our theoretical treatment, we used molecular dynamics simulations to treat Coumarin 152 solvated in dimethylaniline (DMA), calculating the electronic coupling element for electron transfer $\left(H_{\mathrm{DA}}\right)$, using INDO S/CI wave functions ${ }^{45}$ and the Generalized Mulliken-Hush method. ${ }^{46,47}$ More detailed studies concerned with questions about the sensitivity of the coupling to the position of the donor relative to the coumarin may require a more accurate electronic structure theory model. With this in mind, we present results from a series of calculations that address various properties of the ground and excited states of Coumarin 151 (7-amino-4-trifluoromethylcoumarin, CAS no. [53518-15-3]) and Coumarin 120 (7-amino-4-methylcoumarin, CAS no. [26093-31-2]) (see Scheme 1) to assess various methods for treating the electronic structure of these systems, as well as to answer several outstanding questions concerning these two molecules.

In the present study, we focus considerable attention on the application of $\mathrm{DFT}^{48,49}$ and TDDFT ${ }^{50-53}$ to the coumarins. A large body of data has been developed over the past several years to suggest that TDDFT is a powerful tool for the calculation of electronic excitations, particularly for valencelike transitions. ${ }^{54-66}$ To assess the quality of the DFT and TDDFT methods, we have performed a series of electronic structure calculations using several other excited-state approaches. On this basis, we hope to gauge the relative accuracy of the different techniques for describing the electronic structure of the ground and excited states of medium sized organic chromophores such as coumarins. From these results, it becomes clear that accurate excitation energies based on an all-electron treatment demand use of a correlated treatment, but that ZINDO $\mathrm{S} / \mathrm{CI}$ does a good job of describing the excitation energy to the $\mathrm{S}_{1}$ state for both $\mathrm{C} 151$ and $\mathrm{C} 120$. The dipole moment shift upon excitation to the $S_{1}$ state for $\mathrm{C} 151$ is also well described by ZINDO S/CI, but not for C120. In addition, our results suggest that there is likely an error in the current best estimate of the ground-state dipole moment of Coumarin 151, and they also allow us to discriminate between several estimates of the dipole moment shift upon photoexcitation. Finally, we confirm the recent assignment made by Topp and co-workers for their Coumarin 151-water complexes. ${ }^{67-70}$

The rest of the paper is organized as follows. In the following section, we outline the electronic structure methods we have used. In the third section, we present and discuss our results, treating the ground-state equilibrium geometries of C151 and C120. Since limited theoretical treatments of the coumarins have appeared previously, ${ }^{71-77}$ we use these two species as benchmarks for application of several theoretical methods. On the basis of the results of these calculations, we find that TDDFT with any of several hybrid functionals provides excitation energies consistent with the best wave function-based approach we are able to apply (the multi-state CASPT2 method $^{78-80}$ of Roos and co-workers). We then use TDDFT with the B3LYP functional to examine several water-Coumarin 151 complexes, and compare with the results of Topp and co-workers. ${ }^{67-70}$ In the final section we offer our conclusions.

\section{Methods}

A variety of standard basis sets were used in the course of this study including the $6-31 \mathrm{G}(\mathrm{d}),{ }^{81-83} 6-31+\mathrm{G}(\mathrm{d}),{ }^{84} 6-311 \mathrm{G}-$ $(\mathrm{d}, \mathrm{p}),{ }^{85,86} 6-311 \mathrm{G}+(\mathrm{d}, \mathrm{p})$, and the Sadlej polarized (POL) basis sets. ${ }^{87}$ In most cases, only the five $l=2 d$ functions were used, the exceptions being the DFT, CI singles, and RHF calculation which utilized the 6-31G(d) basis; in these cases the six Cartesian $d$ functions were used. No significant differences are expected when comparing results based on use of spherical vs Cartesian $d$ functions.

The ground-state geometries used for C151 and C120 were obtained from optimizations using the B3LYP functional ${ }^{88}$ with the 6-311G(d,p) basis set (geometries provided in Supporting Information). Comparison with optimizations done using AM1 ${ }^{89}$ or B3LYP in the 6-31G(d) basis showed little structural difference, with the exception that the DFT results in either basis produced carbonyl bonds and $\mathrm{C}-\mathrm{F}$ bonds shorter than the AM1 values by approximately $0.02 \AA$. For both molecules, the amine group was noncoplanar with the coumarin ring plane, but the amine protons were symmetrically placed with respect to the ring.

In the case of $\mathrm{C} 151$ we also separately optimized the lowest triplet state $\left(\mathrm{T}_{1}\right)$, and this geometry was obtained based on a B3LYP calculation in the $6-311 \mathrm{G}(\mathrm{d}, \mathrm{p})$ basis. Finally, in the case of C151, we also used CI Singles (CIS) to obtain an estimate for the minimum of the lowest excited singlet state; this optimization was done in the 6-31G(d) basis. To estimate the energy associated with geometrical relaxation for this state, we also reoptimized the ground state of $\mathrm{C} 151$ in the $6-31 \mathrm{G}(\mathrm{d})$ basis using an RHF wave function.

In the TDDFT ${ }^{50-52,58,59,61-63,90}$ calculations, we used the BLYP,${ }^{91,92}$ B3LYP ${ }^{88}$ PBE,${ }^{93,94}$ PBE0,${ }^{54-57,95,96}$ and MPW1PW91 ${ }^{54}$ functionals. Compared to B3LYP, the PBE0 and MPW1PW91 hybrid functionals have previously been shown to yield better agreement with experiment and/or accurate theoretical results over a broad range of states, including low-lying Rydberg transitions. ${ }^{55,56}$ The BLYP and PBE functionals are the only pure Generalized Gradient Approximations (GGAs) used in the present study, and our goal was to assess the importance of the inclusion of exact exchange for the estimation of excitation energies and dipole moments.

None of the qualitative conclusions of this paper depend on whether B3LYP or PBE0 is used for the hybrid functional DFT and TDDFT calculations (see Supporting Information). The former is more popular in quantum chemistry, and often yields more accurate results for systems that it was designed for; the latter is more accurate for solid calculations, ${ }^{97}$ has a nonempirical derivation, and is expected to be more robust. Ongoing calculations for a series of coumarins used even more frequently as solvation probes use the PBE0 functional. ${ }^{98}$

Dipole moments for TDDFT excited states were estimated using a finite field calculation $( \pm 0.001 \mathrm{au})$ and TDDFT excitation energies. Although the Runge-Gross theorem ${ }^{53}$ establishing the validity of the TDDFT approach has only been proven for potentials that decay to zero at infinity, this approach should be adequate for valence-like states because any integrals over the potential are finite due to the use of a truncated Gaussian basis set. A similar approach has been used previously to estimate polarizabilities for excited states of polyenes. ${ }^{99}$ 
Solvent effects on the ground state were examined using two polarizable continuum models, the PCM model of Tomasi et al. ${ }^{100-102}$ and the Onsager polarizable point dipole model. ${ }^{103}$ The former takes account of specific molecular shape in the construction of the solute cavity, whereas the latter assumes a spherical cavity surrounding the solute. Significant differences between ground-state solvation energies and solvated dipole moments are obtained in the two cases, but using an Onsager model we estimate solvation effects on both the ground and excited states for TDDFT, parametrizing the model based on the ground-state PCM calculations (see below). Recent work by the group of Barone and co-workers ${ }^{104-107}$ has led to development of a PCM-like model for TDDFT excited states, but this method is not generally available at present. Nevertheless, the present simple model should include the dominant effects causing the spectral shifts.

All DFT, TDDFT, and CIS calculations, all solvation calculations, and geometry optimizations were performed using Gaussian 98 (versions A.9 and A.11). ${ }^{108}$

Calculations for the ground and excited states of $\mathrm{C} 151$ and C120 were also performed using complete active space SCF theory (CASSCF) and CASPT2 (where second-order perturbation theory corrections are made to the CASSCF energy), in both its single state ${ }^{79,80}$ and multi-state variants. ${ }^{78}$ The multistate approach is needed for the larger reference space calculations we employed because of near-degeneracy in the secondorder energies, and the large reference spaces are used because intruder state effects arise when more modest reference spaces are used. For example, reference space sizes smaller than the 10-electron 10-orbitals space used here for C151 can lead to intruder state problems ${ }^{79}$ wherein the square of the reference space coefficient in the single state CASPT2 calculation was less than 0.1 for one of the states, whereas both other states had values near 0.6. While the excitation energies were not significantly different based on these smaller-reference space calculations (especially for the multi-state CASPT2 results), we nevertheless deemed these less reliable and do not present them here. Similar, but in fact more extreme effects were observed for C120. For C120, we used a 14-electron 10-orbital active space to avoid intruder state effects.

We denote the various CASSCF and CASPT2 calculations in the following way. A CASSCF $n$-state $x / y$ calculation indicates a CASSCF calculation averaging the $n$ lowest electronic states in the CASSCF procedure, distributing $x$ electrons among $y$ orbitals. Single-state (multi-state) CASPT2 calculations based on these CASSCF wave functions are denoted CASPT2 $n$-state $x / y$ (MCASPT2 $n$-state $x / y$ ). Dipole moments are calculated using finite fields for the CASSCF, CASPT2, and MCASPT2 results. No electrons in core orbitals are correlated in the CASPT2 calculations. All CASSCF and CASPT2 calculations were performed using MOLCAS 5.2. ${ }^{109}$ Only MCASPT2 results are presented in the text; CASSCF and CASPT2 results are provided in the Supporting Information.

Due to the size of these systems and the goal of examining a wide range of electron-transfer orientations in the future using simpler approximations, we have also performed calculations using the INDO S/CI method of Zerner and co-workers (ZINDO). ${ }^{45}$ The results presented below are based on the Zerner group code; ${ }^{110}$ calculations using the Gaussian 98 implementation of ZINDO S/CI were also performed (results not shown) and yielded an $S_{1}$ excitation energy within $0.2 \mathrm{eV}$ of that of the Zerner implementation, whereas the $\mathrm{S}_{2}$ state was within $0.4 \mathrm{eV}$ (the Zerner $S_{1}-S_{2}$ splitting was $0.4 \mathrm{eV}$, whereas the Gaussian was $0.2 \mathrm{eV})$.
TABLE 2: Method Dependence of Ground State Dipole Moment for C151 at B3LYP/6-311G(d,p) Optimized Geometry $^{a}$

\begin{tabular}{lll}
\multicolumn{1}{c}{ method } & \multicolumn{1}{c}{ basis } & $\mu(\mathrm{D})$ \\
\hline RHF & $6-311 \mathrm{G}(\mathrm{d}, \mathrm{p})$ & 6.12 \\
MP2 & $6-311 \mathrm{G}(\mathrm{d}, \mathrm{p})$ & 5.5 \\
B3LYP & $6-311 \mathrm{G}(\mathrm{d}, \mathrm{p})$ & 6.22 \\
MCASPT2 4 state avg 10/10 & $6-31 \mathrm{G}(5 \mathrm{~d})$ & 5.9 \\
ZINDO & & 5.86 \\
AM1 & & 5.13 \\
PM3 & $6-311 \mathrm{G}(\mathrm{d}, \mathrm{p})$ & 5.07 \\
B3LYP/PCM/water & $6-311 \mathrm{G}(\mathrm{d}, \mathrm{p})$ & 8.96 \\
RHF/PCM/water &
\end{tabular}

${ }^{a}$ Results with only two significant figures were obtained by numerical differentiation.

\section{Results and Discussion}

In this section, we present results for C151 and C120, organized in the following manner. We first compare various theoretical methods for the description of the ground and excited-state dipole moments and electronic excitation energies at the equilibrium geometry for $\mathrm{C} 151$. We then provide similar results for $\mathrm{C} 120$. Following this, we examine the effects of geometry changes and solvation on the ground and excited states of C151. Finally, we present results for C151-water complexes, examining effects on electronic excitation energies, ionization potentials (IPs), and dipole moments.

A. C151 Ground-State Equilibrium Geometry. i. GroundState Dipole Moment. In Table 2 we present results for the $\mathrm{S}_{0}$ dipole moment of C151. Aside from the AM1 and PM3 results all other methods yield dipole moments in the range of 5.56.4 D. RHF and DFT results tend to be on the higher end of this range $(6.1-6.4 \mathrm{D})$ whereas the MP2 result is on the lower end. The dipole moments based on continuum solvation calculations in Table 2 indicate that the B3LYP solvated dipole moment is enhanced relative to the gas-phase value, significantly more so than the SCF value.

The gas-phase results are weakly dependent on basis set. The TDDFT results for these valence-like states are insensitive to choice of hybrid function (PBE0, MPW1PW91, and B3LYP, see the Supporting Information). Computational constraints required us to use the $6-31 \mathrm{G}(\mathrm{d})$ basis for the MCASPT2 calculations, but the basis set insensitivity of the DFT results suggests that the comparison of MCASPT2 results obtained in the smaller basis with DFT results obtained in larger basis sets should be adequate.

Pure GGA ground state dipole moments are somewhat higher than hybrid values (see Supporting Information), but we find the values roughly satisfy the relation

$$
\mu^{\text {hybrid }}=\mu^{\mathrm{GGA}}+\frac{1}{4}\left(\mu^{\mathrm{RHF}}-\mu^{\mathrm{GGA}}\right)
$$

This relation has a simple origin. Hybrid functionals mix about $1 / 4$ of exact exchange with GGA in order to account for static correlation. ${ }^{111}$ Because the dipole moment yields the total energy change in an electric field, it must satisfy the same relations as ground-state energies. Thus hybrids DO improve dipole moments for the same reason they improve atomization energies, unlike, e.g., ionization potentials. ${ }^{48,112}$ Noting that the dipole moment is a moment of the density, this reasoning also implies that this aspect of the density is improved by going from LSD to GGA to hybrid.

ii. Vertical Electronic Excitation Energies and Excited-State Dipole Moments. In Table 3 we present results for the electronic excitation energies to the first two (or in some cases, three) 
TABLE 3: Basis Set and Method Dependence of Excitation Energies for B3LYP/TDDFT Results for C151 at Optimized B3LYP/6-311G(d,p) Geometry

\begin{tabular}{|c|c|c|c|c|}
\hline method & basis & $\Delta E_{1}(\mathrm{eV})$ & $\Delta E_{2}(\mathrm{eV})$ & $\Delta E_{3}(\mathrm{eV})$ \\
\hline B3LYP/TDDFT & $6-311 G(d, p)$ & 3.73 & 4.19 & 4.38 \\
\hline CIS & $6-311 \mathrm{G}(\mathrm{d}, \mathrm{p})$ & 4.90 & 5.66 & 6.15 \\
\hline $\begin{array}{l}\text { MCASPT2 } \\
\quad \text { 4-state } 10 / 10^{a}\end{array}$ & $6-31 G(d)$ & 3.46 & 4.46 & \\
\hline ZINDO S/CI & & 3.84 & 4.22 & 4.26 \\
\hline
\end{tabular}

TABLE 4: Excited State Dipole Moment and Angle Differences Relative to Ground State for C151 at B3LYP/ 6-311G(d,p) Optimized Geometry

\begin{tabular}{lccc}
\hline \multicolumn{1}{c}{ method } & basis & $\Delta \mu_{1}(\mathrm{D}) / \Delta \theta(\mathrm{deg})$ & $\Delta \mu_{2}(\mathrm{D}) / \Delta \theta(\mathrm{deg})$ \\
\hline B3LYP/TDDFT & $6-311 \mathrm{G}(\mathrm{d}, \mathrm{p})$ & $4.6 / 8.9$ & $4.0 / 11.3$ \\
CIS & $6-311 \mathrm{G}(\mathrm{d}, \mathrm{p})$ & $3.1 / 7.3$ & $1.2 / 5.0$ \\
ZINDO S/CI & & $3.0 / 13.8$ & $2.3 / 4.9$ \\
MCASPT2 & $6-31 \mathrm{G}(5 \mathrm{~d})$ & $6.6 / 8.0$ & $0.1 / 1.0$
\end{tabular}

${ }^{a}$ Excitations from 18 occupied orbitals into all virtuals.

singlet excited states at the ground-state equilibrium geometry for $\mathrm{C} 151$. The CIS results are $1.2-1.5 \mathrm{eV}$ higher than the TDDFT results, as has been observed in a variety of other studies of aromatic molecules. ${ }^{61-63}$ The ZINDO S/CI results are in good agreement with the TDDFT results, both for $S_{1}$ and $\mathrm{S}_{2}$. The CASSCF excitation energies are higher than the TDDFT results (see the Supporting Information), but the MCASPT2 excitation energies (either single-state or multi-state) are much closer to the TDDFT results.

The TDDFT excitation energies depend only weakly on basis set, with addition of diffuse functions lowering the excitation energies by approximately $0.1 \mathrm{eV}$, and other basis set variations having smaller effects (Supporting Information). Other choices of exchange-correlation functional are also examined in data presented in the Supporting Information, and it is found that all hybrid functionals examined yield similar excitation energies, whereas the pure GGAs produce excitation energies lower than the TDDFT results of Table 3 by approximately $0.5 \mathrm{eV}$.

In Table 4, excited state dipole moment differences are presented based on TDDFT, CIS, MCASPT2, and ZINDO S/CI results. The TDDFT excited state dipole moment differences are relatively insensitive to basis set and hybrid functional choice (see the Supporting Information), whereas the pure GGAs tend to yield somewhat larger dipole moment changes. The CIS and ZINDO dipole moment changes are about $1-1.5 \mathrm{D}$ below those obtained using TDDFT. The multi-state CASPT2 approach yields a large dipole moment for the $S_{1}$ state, and a much smaller value for the $S_{2}$ state. Given the second-order treatment, the near-degeneracy of the pair of interacting states, and the large variation in dipole moments between CASPT2 and MCASPT2 (see the Supporting Information) the MCASPT2 values are not likely to be converged results. However, the series of calculations does point to the importance of correlation and multistate effects on the dipole moment computed using CASSCF/ CASPT2.

iii. Comparison of Experimental and Theoretical GroundState Dipole Moments. We postpone comparison of excitation energies with experiment until section III C, where geometrical relaxation effects are discussed. At this point, we can compare our results with previous ground-state dipole moments. ${ }^{72,113-115}$ Moylan's experimental ground-state dipole moment for C151 is $4.59 \mathrm{D}$, measured in $\mathrm{CHCl}_{3}$ solution. ${ }^{113}$ With the exception of the semiempirical methods (AM1 and PM3), all of our theoretical results give dipole moments higher than this value by $1-1.6 \mathrm{D}$. All of these dipole moment estimates are based on calculations performed at the B3LYP optimized geometry. The previous AM1 results ${ }^{72}$ yield ground-state dipole moments in good agreement with our DFT results, while the PM3 results of Moylan ${ }^{113}$ and others ${ }^{69,70,114,115}$ are significantly lower than our results. For all other coumarins studied by Moylan, the PM3 results are markedly lower than the experimental values, while in the case of C151 they are in excellent agreement. ${ }^{113}$

One might attempt to argue that the discrepancy between our results and experiment could be attributed to inadequacies in method and/or basis set, but the range of methods employed here, along with the basis set insensitivity (even up to use of the Sadlej basis) suggests that our result is robust. It has been previously observed that for polymeric systems, DFT yields corrections to the RHF polarizability that are too large and of the wrong sign. ${ }^{99}$ This problem may result in a tendency to overestimate the ground-state dipole moment here as well, but we do not expect the error to be large enough to support the experimental value of 4.59 D, given our MP2 value.

The dipole moment is sensitive to the ground-state geometry. To assess this sensitivity, we have performed ground-state geometry optimizations in the 6-31G(d) basis at the RHF level of theory, and used this geometry to estimate both SCF (6-31G(d): 5.7D) and B3LYP (6-311G(d,p): 5.8 D) dipole moments. Although these are somewhat closer to the results of Moylan, they are still over 1D too high. Geometry optimization of $\mathrm{C} 151$ at the PM3 level yields ring-N-H dihedral angles that are $10^{\circ}$ larger than those for the AM1 or B3LYP methods (2830 vs $18-20$ degrees, respectively), leading to a PM3 geometry that has a substantially more pyramidal nitrogen geometry than the other methods. (The PM3 normal-mode analysis also yields an unrealistically large frequency for the inversion coordinate.) Using the ground-state geometry from a PM3 geometry optimization we obtain ground-state dipole moments of 4.43 D, 4.59 D, 5.74 D, 5.81 D for PM3, AM1, RHF(6-311G(d,p)), and B3LYP (6-311G(d,p)), respectively. Our PM3 value agrees with the previous PM3 value, ${ }^{113}$ but the RHF and B3LYP values are significantly higher. The MP2 dipole moment based on an MP2 geometry optimization for $\mathrm{C} 151$ in the 6-311G(d,p) basis (5.14D) is similar to the MP2 dipole moment at the B3LYP geometry in the same basis, so it is unlikely that the B3LYP geometry is grossly in error. The MP2 dipole moment based on the optimized geometry using the $6-311 \mathrm{G}(\mathrm{d}, \mathrm{p})$ basis is within $0.6 \mathrm{D}$ of the experimental value, but results in the Supporting Information indicate that diffuse functions will tend to increase, not decrease, this value.

It is possible that the solution geometry is different from the gas-phase geometry obtained via the calculations discussed above. In particular, it is possible that there is relatively free rotation about the amine ring bond in solution. We performed a series of calculations in which the amine was rotated about the amine-ring bond, maintaining all other bond lengths and angles at the values in the equilibrium geometry. Defining the equilibrium geometry as $0^{\circ}$, and the geometry corresponding to the ring plane bisecting the $\mathrm{H}-\mathrm{N}-\mathrm{H}$ angle of the amine as $90^{\circ}$, we found that the ground-state dipole moment (B3LYP/ $6-311 \mathrm{G}(\mathrm{d}, \mathrm{p}))$ varied little up to an angle of $45^{\circ}\left(6.22 \mathrm{D}\right.$ at $0^{\circ}$, $5.81 \mathrm{D}$ at $45^{\circ}$ ), but at $60^{\circ}$, the ground-state dipole moment dropped to $4.83 \mathrm{D}$, similar to the value obtained by Moylan. ${ }^{113}$ The energy of the $60^{\circ}$ geometry is $8.1 \mathrm{kcal} / \mathrm{mol}$ higher than the equilibrium geometry which, whereas somewhat high, may be varied in solution to the point where geometries such as these 
TABLE 5: Method Dependence of Excitation Energies for C120 at C120 Optimized B3LYP/6-311G(d,p) Geometry

\begin{tabular}{llccc}
\hline \multicolumn{1}{c}{ method } & \multicolumn{1}{c}{ basis set } & $\Delta E_{1}(\mathrm{eV})$ & $\Delta E_{2}(\mathrm{eV})$ & $\Delta E_{3}(\mathrm{eV})$ \\
\hline B3LYP/TDDFT & 6-311G(d,p) & 4.00 & 4.45 & 4.60 \\
CIS & 6-311G(d,p) & 5.09 & 5.71 & 6.39 \\
MCASPT2 & 6-31G(5d) & 3.78 & 4.63 & \\
$\quad$ 3 state 14/10 & & & & \\
ZINDO & 6-31G(5d) & 3.88 & 4.24 &
\end{tabular}

might be significant contributors to the thermally averaged dipole moment. Nibbering, et al. ${ }^{24-26}$ have shown that chloroform (the solvent used in the work by Moylan ${ }^{113}$ for dipole and hyperpolarizability measurements on coumarins) is an H-bond donor to the carbonyl oxygen of $\mathrm{C} 102$, a related 7-amino coumarin. It may also be that unusual effects arise for the case of $\mathrm{C} 151$ because of similar specific solvation.

Overall, it appears that RHF and DFT tend to overestimate the ground state dipole moment of C151. However, unless the rotation about the amine bond is relatively free in solution, the ground state dipole moment is consistently higher than the experimental value of Moylan (measured in $\left.\mathrm{CHCl}_{3}\right) .{ }^{113}$ Our solvation results in Table 2 show that presence of a dielectric reaction field enhances the dipole moment of $\mathrm{C} 151$. Thus, we would expect the experimental value for the dipole moment to be larger than our calculated value, indicating that reexamination of the C151 dipole moment may be warranted.

iv. Comparison of Excited-State Dipole Moments for C151 with Previous Results. Comparison of the results of Tables 2 and 4 with the results of other workers in Table 1 indicates that our CIS, ZINDO S/CI, and TDDFT vector dipole moment differences fall in the middle of the range of $S_{1}-S_{0}$ state dipole moment differences $(1.7-6.3 \mathrm{D})$ and are in good agreement with the results of Fessenden et al. ${ }^{8}$ One would expect solvation to increase the dipole moment difference if the excited state is more polarizable than the ground state, so our gas-phase values should be viewed as lower bounds for theoretical solution-phase dipole moment differences, although the polarizability difference between the ground and excited states is modest. On the other hand, it is possible that the errors inherent in using DFT for dipole moments for the coumarins effectively cancel for dipole moment differences, thus leading to reasonable estimates of these differences. The best agreement with experiment for our calculated gas phase dipole difference occurs for C151 in benzene, which will exhibit solvation due to quadrupolar interactions, but should not dramatically polarize the two states, indicating that the dipole moment difference is probably reasonable. The solution-phase excitation energies presented below suggest that the TDDFT dipole moment differences may be somewhat high, and that a more accurate value is between the CIS and TDDFT results. The difference between the two methods is on the order of $1 \mathrm{D}$, so either method appears to yield reasonable excited-state dipole moments.

B. C120 Ground-State Equilibrium Geometry. In Tables 5 and 6, we present results for $\mathrm{C} 120$ at its ground state equilibrium geometry that parallel those presented for C151. In particular, we examine the method dependence of the excitation energies (Table 5) and ground and excited state dipole moments (Table 6). (Basis set and more complete methods comparisons are presented in the Supporting Information.) Quite similar conclusions can be drawn for $\mathrm{C} 120$ based on these results to those that were obtained for C151. In particular, (i) the ground state dipole moment is weakly dependent on basis set, exhibits a range of values as a function of theoretical method, and tends to be somewhat larger than that for $\mathrm{C} 151$, (ii) the electronic excitation energies at the TDDFT level of theory are weakly
TABLE 6: Method Dependence of Ground and Excited State Dipole Moments for C120 at B3LYP/6-311G(d,p) Optimized Geometry

\begin{tabular}{llccc}
\hline \multicolumn{1}{c}{ method } & \multicolumn{1}{c}{ basis } & $\mu_{\mathrm{S} 0}(\mathrm{D})$ & $\begin{array}{c}\mu_{\mathrm{S} 1}(\mathrm{D}) / \Delta \theta \\
(\mathrm{deg})^{a}\end{array}$ & $\begin{array}{c}\mu_{\mathrm{S} 2}(\mathrm{D}) / \Delta \theta \\
(\mathrm{deg})^{a}\end{array}$ \\
\hline SCF & 6-311G(d,p) & 6.9 & & \\
MP2 & 6-311G(d,p) & 5.6 & & \\
B3LYP/TDDFT & 6-311G(d,p) & 6.4 & $9.1 / 18.6$ & $8.2 / 18.1$ \\
CIS & 6-311G(d,p) & 6.9 & $8.1 / 12.3$ & $7.05 / 4.6$ \\
MCASPT2 $^{\quad 6-31 G(5 d)}$ & 6.1 & $8.6 / 24.0$ & $7.2 / 13.1$ \\
$\quad$ 3 state 14/10 & & & & \\
ZINDO $^{b}$ & & 7.1 & $7.0 / 14.0$ & $8.4 / 7.6$ \\
AM1 & & 5.6 & & \\
PM3 & & 5.4 & &
\end{tabular}

${ }^{a}$ For the excited states the first entry is the dipole moment, the second is the angle between the ground state and excited-state dipole moments. ${ }^{b}$ Excitations from 16 occupied orbitals into all virtual orbitals.

dependent on basis set and hybrid functional, and are in reasonable agreement with the ZINDO and MCASPT2 results, (iii) the CIS and CASSCF excitation energies are too high, (iv) the excited-state dipole moment differences tend to be somewhat smaller for the CIS and CASSCF results than those based on TDDFT, and (v) the TDDFT excited-state dipole moment differences fall in the middle of the range of the experimental values. Unlike C151, the ZINDO S/CI dipole moment for the $S_{1}$ state is considerably smaller than that for any of the other methods.

As was the case for C151, we find that our calculated groundstate dipole moments are larger than the experimental results (Nemkovich et al.). ${ }^{4,5}$ Although the MP2 value for the $\mathrm{C} 120$ dipole moment is similar to the experimental value in dioxane, the measured dipole moment is expected to be enhanced in solution, as noted above. Thus, on the basis of the experimental solution phase dipole moment, the gas-phase value would be expected to be significantly smaller than our calculated values. Rotation about the amine-ring bond may lead to lower thermally averaged dipole moments in solution, as we suggested above for C151. However, it is also possible that the Nemkovich et al. values are artificially low. Their ground-state dipole moments were obtained in the course of electrooptical studies of the dipole moment difference between the ground and excited state, and are based on the assumption of collinear ground and excitedstate dipoles. They also neglect polarizability contributions to the electrical response. Our results suggest these assumptions may not be valid, and may be responsible for an underestimate of the C120 ground-state dipole moment.

On the basis of comparisons of the $\mathrm{C} 120$ and $\mathrm{C} 151$ absorption maxima (solution, $0.3 \mathrm{eV}$ difference) ${ }^{72}$ and the similarity of the dipole moments in these systems, it would appear that the experimental difference in $\mathrm{S}_{1}$ vertical excitation energies is approximately $0.35 \mathrm{eV}$. The theoretical estimates are: TDDFT $=0.25-0.3 \mathrm{eV}, \mathrm{ZINDO} \mathrm{S} / \mathrm{CI}=0.0 \mathrm{eV}, \mathrm{CIS}=0.2 \mathrm{eV}, \mathrm{CAS}-$ $\mathrm{SCF}=0.6 \mathrm{eV}, \mathrm{CASPT} 2=0.1 \mathrm{eV}, \mathrm{MCASPT} 2=0.32 \mathrm{eV}$.

C. Geometrical Relaxation Effects for C151. In this section, we address several types of relaxation effects on the excitation energy for $\mathrm{C} 151$. We consider the following: (i) rigid rotation about the amine-ring bond, to assess the likelihood of TICTformation for $\mathrm{C} 151$, (ii) the effects of motion along an inversionlike coordinate at the amine, (iii) energy changes in the ground and $S_{1}$ state using estimated equilibrium geometries for the $S_{1}$ state, and (iv) solvation effects on the spectrum using a dielectric continuum model by Kim. ${ }^{116,117}$

i. Rotation about the Amine-Ring Bond. In Table 7, we examine the effects of rigid rotation of the amine group of $\mathrm{C} 151$ on the $S_{1}$ and $S_{2}$ excitation energies. Specifically, this rigid rotation entails preservation of the $\mathrm{H}-\mathrm{N}-\mathrm{H}$ bond angle and 
TABLE 7: Ground and Excited State Relative Energies vs Rigid Twist Angle from C151 6-311G(d,p) B3LYP Optimized Geometry ${ }^{a}$

\begin{tabular}{cccc}
\hline angle $(\mathrm{deg})$ & $\Delta E_{\mathrm{S} 0}(\mathrm{kcal} / \mathrm{mol})$ & $\Delta E_{\mathrm{S} 1}(\mathrm{kcal} / \mathrm{mol})$ & $\Delta E_{\mathrm{S} 2}(\mathrm{kcal} / \mathrm{mol})$ \\
\hline \multicolumn{4}{c}{ no field } \\
15 & 0.63 & 0.60 & 0.17 \\
30 & 2.45 & 2.42 & 1.08 \\
45 & 5.15 & 5.11 & 2.54 \\
60 & 8.17 & 7.80 & 3.77 \\
75 & 10.67 & 9.91 & 4.15 \\
90 & 11.71 & 10.00 & 3.33 \\
& & & \\
15 & 0.62 & & \\
30 & 2.64 & 2.22 & 0.23 \\
45 & 5.73 & 4.64 & 1.29 \\
60 & 9.28 & 6.40 & 3.02 \\
75 & 12.30 & 5.23 & 5.02 \\
90 & 20.55 & 32.8 & 6.68 \\
& & &
\end{tabular}

${ }^{a}$ Energies relative to value at equilibrium geometry. Energies calculated using B3LYP for the ground state, and B3LYP/TDDFT for the excited states, in the $6-311 \mathrm{G}(\mathrm{d}, \mathrm{p})$ basis.

both $\mathrm{H}-\mathrm{N}-\mathrm{C}$ bond angles, while twisting about the $\mathrm{N}-\mathrm{C}$ bond. The angle zero degrees is defined as the equilibrium geometry from the B3LYP optimization. It is seen that the $S_{1}$ and $S_{2}$ states show no energetic preference toward amino group twisting at this level of theory (ground state, B3LYP; excited-state B3LYP/ TDDFT, both 6-311G(d,p)), indicating that in the gas-phase, TICT state formation is not expected for C151. In TDDFT studies of DMABN in the gas phase ${ }^{118}$ it was shown that TDDFT tended to exaggerate the tendency to yield a TICTlike state, underestimating the barrier to form the TICT state and overestimating its stability. Thus, the fact that we do not observe a stable TICT state is strong evidence that one should not expect it to be observed in the gas-phase.

Though not predicted to occur in the gas-phase, a TICT state could exist in solution where polar solvation effects may enhance the stability of a TICT state. To address solvation effects, we have performed the twist calculations with a constant applied electric field intended to approximate the solvent reaction field in water with respect to the ground state (field based on an Onsager calculation using a solute cavity radius $=4.73 \AA$, $\epsilon=$ 78.39, using B3LYP/6-311G(d,p)). This calculation is relatively crude, in that it neglects the geometrical dependence of the reaction field as well as the electronic response of the dielectric field to the excited state dipole moment. When nonequilibrium solvent methods become generally available for the description of TDDFT excited states ${ }^{104-107}$ improved estimates can be made. For the present, we expect that the qualitative aspects of the solvent contributions will be included in these calculations, and should give a reasonable assessment of whether solvent will produce a TICT state for C151. In the second portion of Table 7 , it is seen that the solvent reaction field does not alter the conclusions drawn from the case with no field present, i.e., TICT state formation is not predicted in $\mathrm{C} 151$.

ii. Effects Due to Inversion at the Amine. In Table 8, we examine the effects of inversion at the amine group. These calculations were performed by symmetrically changing the dihedral angles involving the $\mathrm{H}-\mathrm{N}$ bonds, relative to the values in the ground state equilibrium structure (the initial equilibrium geometry is also slightly modified from the original optimized geometry, to symmetrize these two angles. The energy and dipole moment at this new equilibrium geometry are essentially identical to the original equilibrium geometry). All other coordinates are fixed in the process. It is seen that the $S_{1}$ state tends to be somewhat more planar than the ground state, whereas
TABLE 8: Ground and Excited State Relative Energies vs Inversion Coordinate from C151 6-311G(d,p) B3LYP Optimized Geometry ${ }^{a}$

\begin{tabular}{cccc}
\hline angle $(\mathrm{deg})$ & $\Delta E_{\mathrm{S} 0}(\mathrm{kcal} / \mathrm{mol})$ & $\Delta E_{\mathrm{S} 1}(\mathrm{kcal} / \mathrm{mol})$ & $\Delta E_{\mathrm{S} 2}(\mathrm{kcal} / \mathrm{mol})$ \\
\hline 0 & 1.020 & 0.30 & 1.31 \\
5 & 0.911 & 0.23 & 1.19 \\
10 & 0.566 & 0.04 & 0.78 \\
15 & 0.164 & -0.10 & 0.27 \\
25 & 0.461 & 0.99 & 0.26 \\
35 & 4.982 & 6.50 & 4.45
\end{tabular}

${ }^{a}$ Energies relative to value at equilibrium geometry, which corresponds to an angle of $18.73^{\circ}$. Energies calculated using B3LYP for the ground state, and B3LYP/TDDFT for the excited states, in the 6-311G $(\mathrm{d}, \mathrm{p})$ basis.

the opposite is true for the $\mathrm{S}_{2}$ state. Furthermore, the potential energy surface of the $S_{1}$ state is much flatter near the planar geometry. These results are in qualitative agreement with the suggestion of Pal, et al. based on their gas-phase results for C151 which suggest that in the $S_{1}$ state, inversion is more likely than in the ground state. ${ }^{119}$ We find that the dipole moment difference between the $S_{1}$ and $S_{0}$ states is $4.7 \mathrm{D}$ at the planar geometry, which is similar to that obtained at the ground state equilibrium geometry (Table 4). It is certainly possible that with solvent present and/or at a different level of theory, the $\mathrm{S}_{1}$ state might actually be found to be planar at the amine.

iii. Energetic Changes in $S_{1}$ and $S_{0}$ Using Estimated $S_{1}$ Equilibrium Geometries. Our calculated excitation energies to this point correspond to vertical absorptions (see Figure 1A) because they are performed at the optimized geometry for the ground state of the systems. Neglecting zero-point energies, our TDDFT excitation energies at the $\mathrm{S}_{0}$ geometry therefore correspond to vertical absorption lines. The vertical transition from the ground state is frequently (but not always) associated with the maximum in the absorption spectra, thus leading to some ambiguity in comparisons between theoretical and experimental transition energies. A somewhat less ambiguous comparison would be the $0-0$ transition (or, neglecting zeropoint effects the energy difference between a pair of states calculated at their respective equilibrium geometries). Thus, to calculate the $0-0$ absorption line we would need both the $S_{0}$ and the $\mathrm{S}_{1}$ equilibrium geometries.

We are unable to perform a geometry optimization of the excited singlet states at the TDDFT level of theory, since an implementation of gradients for TDDFT is not available at this time. However, to obtain an estimate of the geometrical relaxation energy for the $S_{1}$ state as it proceeds from the groundstate geometry to that of the equilibrium $S_{1}$ geometry, we have used two schemes, each of which makes reference to the quantities noted in Figure 1. In either case we calculate the 0-0 transition energy as

$$
\Delta E_{0-0}=\Delta E_{\mathrm{S} 1-\mathrm{S} 0}\left(X_{\mathrm{S} 0}\right)-\Delta E_{\mathrm{S} 1}^{\mathrm{reorg}}
$$

where

$$
\Delta E_{\mathrm{S} 1}^{\mathrm{reorg}}=E_{\mathrm{S} 1}\left(X_{\mathrm{S} 0}\right)-E_{\mathrm{S} 1}\left(X_{\mathrm{S} 1}\right)
$$

and where $X_{\mathrm{S} 0}$ and $X_{\mathrm{S} 1}$ indicate the equilibrium geometries for the $S_{0}$ and $S_{1}$ states, respectively. $E_{\mathrm{S} 1}\left(X_{\mathrm{S} 0}\right)$ and $E_{\mathrm{S} 1}\left(X_{\mathrm{S} 1}\right)$ denote the energies for the $S_{1}$ state calculated at $X_{S 0}$ and $X_{S 1}$, respectively. In a similar fashion, one can estimate the fluorescence maximum as the vertical transition from the $S_{1}$ equilibrium geometry, yielding

$$
\Delta E_{\mathrm{vert}}\left(X_{S 1}\right)=\Delta E_{0-0}-\Delta E_{\mathrm{S} 0}^{\mathrm{reorg}}
$$




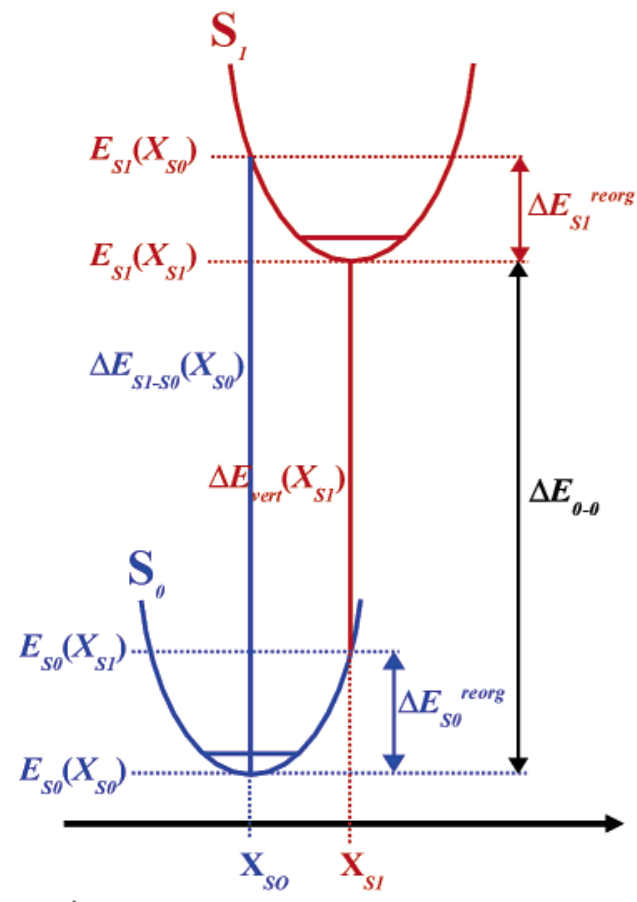

A.

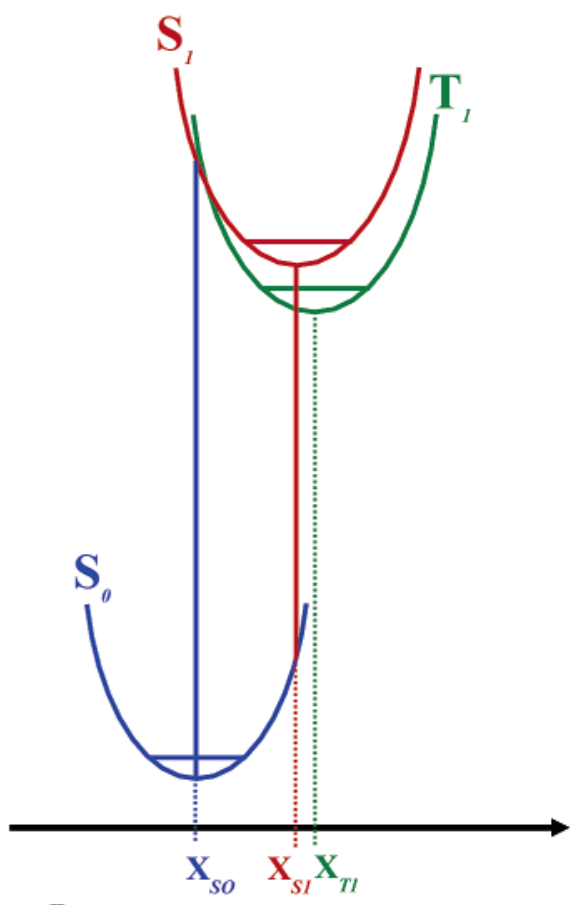

B.

Figure 1. (A) Schematic potential energy surfaces for ground and first excited states of aminocoumarins, illustrating the energies and displacements used in eqs 1-4. (B) Schematic PESs illustrating assumed relative displacements between first excited singlet and triplet states, used in triplet approximation.

TABLE 9: Estimated 0-0 and Fluorescence Maxima for $\mathrm{C151}^{a}$

\begin{tabular}{lccccc}
\hline scheme & $\begin{array}{c}\Delta E_{\text {vert }}\left(\mathrm{S}_{0}\right) \\
(\mathrm{eV})\end{array}$ & $\begin{array}{c}\Delta E_{\mathrm{S} 1}^{\text {reorg }} \\
(\mathrm{eV})\end{array}$ & $\begin{array}{c}\Delta E_{\mathrm{S} 0}^{\text {reorg }} \\
(\mathrm{eV})\end{array}$ & $\begin{array}{c}\Delta E_{0-0} \\
(\mathrm{eV})\end{array}$ & $\begin{array}{c}\Delta E_{\text {vert }}\left(\mathrm{S}_{1}\right) \\
(\mathrm{eV})\end{array}$ \\
\hline triplet & 3.73 & 0.04 & 0.31 & 3.69 & 3.38 \\
CIS & 3.73 & 0.29 & 0.08 & 3.44 & 3.36
\end{tabular}

${ }^{a}$ Quantities defined in Section III C iii.

where

$$
\Delta E_{\mathrm{S} 0}^{\mathrm{reorg}}=E_{\mathrm{S} 0}\left(X_{\mathrm{S} 1}\right)-E_{\mathrm{S} 0}\left(X_{\mathrm{S} 0}\right)
$$

The difference between the two schemes is the method used to calculate the $S_{0}$ and $S_{1}$ reorganization energies.

In the first scheme (denoted "triplet" in Table 9), we used the geometry of the $T_{1}$ state for $\mathrm{C} 151$ as an estimate of the $S_{1}$ equilibrium geometry. The geometry optimization for the $T_{1}$ state was done at the UB3LYP/6-311G(d,p) level. Given that both the $\mathrm{S}_{1}$ and $\mathrm{T}_{1}$ states are largely HOMO $\rightarrow$ LUMO in character, we reasoned that their equilibrium geometries would be similar; this approximation is illustrated schematically in Figure 1B. The results for various excitation energies based on approximating the $S_{1}$ equilibrium geometry using the $T_{1}$ state are shown in Table 9. The estimated $0-0$ transition energy is about $0.04 \mathrm{eV}$ lower than the calculated absorption maximum, and about $0.14 \mathrm{eV}$ higher than the experimental $0-0$ transition. ${ }^{67}$ In this scheme, the ground state energy shifts significantly more for the $S_{0}$ to $S_{1}$ geometry change than does the $S_{1}$ state.

In the second scheme, denoted "CIS" we used a CIS optimization in the $6-31 \mathrm{G}(\mathrm{d})$ basis to estimate the $\mathrm{S}_{1}$ state equilibrium geometry. It is inappropriate to use energy differences based on this geometry together with the B3LYP/6-311G$(\mathrm{d}, \mathrm{p})$ ground-state geometry to calculate values for $\Delta E^{\text {reorg }}$ because the CIS calculation includes no electron correlation, and the calculated reorganization energy would contain contributions from both state changes (i.e., $\mathrm{S}_{1} \leftarrow \mathrm{S}_{0}$ ) and differences in theoretical method (CIS, much like RHF, should yield bonds that are too short compared with B3LYP). Thus, to calculate reorganization energies (based to the extent possible) on only those contributions that arise from state changes, we performed an RHF/6-31G(d) optimization of the ground state. We then used B3LYP or TDDFT/B3LYP to calculate the $S_{0}$ and $S_{1}$ energies at the RHF/S $/ \mathrm{S}_{0}$ and $C I S / \mathrm{S}_{1}$ geometries, calculating the two reorganization energies based on these total energies. The reorganization energies obtained in this fashion are then combined with the TDDFT vertical absorption energy based on the B3LYP/6-311G(d,p) geometry to estimate $\Delta E_{0-0}$ and $\Delta E_{\mathrm{vert}}\left(S_{1}\right)$. In this case the calculated $0-0$ energy is $3.44 \mathrm{eV}$, about $0.11 \mathrm{eV}$ lower than the experimental value.

Comparing the Triplet and CIS schemes, we find that the 0-0 transitions differ by about $0.25 \mathrm{eV}$. The two fluorescence maxima are in excellent agreement with each other, but this is somewhat fortuitous, in that in the Triplet case the ground state reorganization energy is significantly larger than that for the $S_{1}$ state, whereas using the $S_{1}$ CIS minimum, the reverse is true.

Of course, neither of these estimates is the final word on the $\mathrm{S}_{1}$ state equilibrium geometry, and thus neither estimate of the $0-0$ absorption energy could be definitive. Indeed, there are several other factors that may give rise to potential errors in this calculation. The basis set, although of respectable size for a molecule of this type, is by no means sufficient to ensure 0.1 $\mathrm{eV}$ accuracy for excited states in general. The results presented above suggest that the $S_{1}$ state excitation energy is relatively insensitive to basis set expansion, but diffuse functions would contribute to an energy lowering of about $0.1 \mathrm{eV}$, for example. In addition, we have neglected zero-point corrections which, though small will not be zero for these two states. Finally, previous results based on TDDFT suggest that it is a useful spectroscopic tool for valence-like states, but is certainly not expected to yield accuracies at the $0.1 \mathrm{eV}$ level in general. ${ }^{61-63}$ Indeed, it is known that as system size increases TDDFT 
energies go over to Kohn-Sham excitations, which yield significant errors for solid-state band gaps. ${ }^{99}$

Despite these cautions with respect to our calculated excitation energies, the TDDFT results are in remarkably good agreement with experiment, whichever scheme is used to estimate the $0-0$ transition energy. In either case, the reorganization energy of the $S_{1}$ state is modest, and one would expect the vertical and 0-0 transition energies to be similar. Thus, on the basis of these results, it would appear that the TDDFT vertical excitation energies should be consistent with experimental vertical excitations. In comparison with CIS or CASSCF the improvement in excitation energies is dramatic. MCASPT2 results are of similar quality to the TDDFT results, but the questions associated with intruder state effects and the size of the calculation significantly limit the general application of CASPT2 to coumarins, although it will serve as an important benchmark and consistency check for TDDFT on these systems, especially should double excitations become important for certain states.

iv. Solvent Shift Calculations for C151. In this section, we use the above B3LYP/TDDFT results in the 6-311G(d,p) basis to estimate absorption maxima for $\mathrm{C} 151$ in a variety of solvents, for comparison with the results of Pal and co-workers. ${ }^{119}$ All of the provisos discussed in the previous section concerning the accuracy of the calculations presented there apply here, in addition to the uncertainties associated with the solvation model, so we do not expect quantitative accuracy. The solvation model is a means of checking the dipole moment change for the $S_{1}$ state (the term including which is the largest contributor to the solvation energy), and to understand trends in the experimental data. As will be shown below, the results are accurate enough to suggest that specific solvation (H-bonding) has a quantifiable effect on the solution spectroscopy. Nevertheless, it should be noted that we treat solvent-induced mixing of the adiabatic states only perturbatively, and the coupling of solvent and vibrational nuclear modes (potentially important for intense transitions in solution) is neglected. Higher order solvent-induced mixing, and inclusion of the solvent-vibration coupling can lead to solventdependent spectral widths as well as (more modest) modifications to the excitation energy, as has been discussed by Matyushov and Newton. ${ }^{76}$

To predict the solvent shift expected for the spectra of C151, we have estimated solvent shifts using a cavity dielectric continuum model developed by Kim, assuming a spherical solute cavity embedded in a dielectric continuum. ${ }^{16,117}$ In this model, the solvent electronic polarization is treated quantum mechanically rather than classically, as in several other previous treatments. ${ }^{116,117}$ This leads to a somewhat lower solvent electronic response to the solute. However, this is a modest effect in the present calculations. We have calculated the solvent shift of absorption maxima, using eq 3.6 from $\mathrm{Kim}^{116,117}$

$$
\begin{aligned}
\Delta G_{\mathrm{S} 0}^{\mathrm{S} 1}= & -R_{\mathrm{or}}^{\mathrm{S} 0} \Delta \mu\left(1+\alpha_{\mathrm{av}} R_{\mathrm{eq}}^{\mathrm{S} 0}\right) \mu_{\mathrm{S} 0}-\frac{1}{2} R_{\infty}^{\mathrm{S} 0}\left(\Delta \mu^{2}+\Delta M^{2}\right)+ \\
& \frac{1}{2}\left(R_{\mathrm{or}}^{\mathrm{S} 0}\right)^{2} \mu_{\mathrm{S} 1} \Delta \alpha \mu_{\mathrm{S} 0}-\frac{1}{2} R_{\mathrm{or}}^{\mathrm{S} 0}\left(2 R_{\mathrm{or}}^{\mathrm{S} 0}+R_{\infty}^{\mathrm{S} 0}\right) \mu_{\mathrm{S} 0} \Delta \alpha \mu_{\mathrm{S} 0}- \\
& \frac{1}{8}\left(R_{\infty}^{\mathrm{S} 0}\right)^{2} \Delta \mu \alpha_{\mathrm{av}} \Delta \mu-\frac{1}{16}\left(R_{\infty}^{\mathrm{S} 0}\right)^{2} \mu_{\mathrm{S} 1} \Delta \alpha \mu_{\mathrm{S} 1}- \\
& \frac{1}{16}\left(R_{\infty}^{\mathrm{S} 0}\right)^{2} \mu_{\mathrm{S} 0} \Delta \alpha \mu_{\mathrm{S} 0}
\end{aligned}
$$

where $\Delta \mu$ is the dipole difference vector, $\Delta \alpha$ is the difference of polarizability tensors for the two states, and $\alpha_{a v}$ is the average of the polarizability tensors for the two states. The $R_{x}^{y}$ are reaction field factors, with $x$ denoting the solvent polarization
TABLE 10: Estimates of C151 Vertical Absorption Maxima in Various Solvents ${ }^{a}$

\begin{tabular}{lcccc}
\hline \multicolumn{1}{c}{ solvent } & $\epsilon_{\mathrm{o}} / n^{2}$ & $\begin{array}{c}\lambda_{\text {abs max TDDFT }^{b}} \\
(\mathrm{~nm})\end{array}$ & $\begin{array}{c}\lambda_{\text {abs max CIS }}{ }^{c} \\
(\mathrm{~nm})\end{array}$ & $\begin{array}{c}\text { expt }^{d} \\
\lambda_{\text {abs max }} \\
(\mathrm{nm})\end{array}$ \\
\hline gas-phase & & 333 & $(333)^{a}$ & \\
hexane & $1.89 / 1.89$ & $357 / 347$ & $353 / 339$ & 347 \\
cyclohexane & $2.02 / 2.03$ & $358 / 348$ & $354 / 339$ & 348 \\
acetonitrile & $37.5 / 1.81$ & $374 / 364$ & $363 / 349$ & 367 \\
MeOH & $32.7 / 1.76$ & $374 / 364$ & $363 / 349$ & 378 \\
OcOH & $10.3 / 2.04$ & $373 / 361$ & $363 / 347$ & 383 \\
DMSO & $46.6 / 2.19$ & $378 / 365$ & $366 / 350$ & 386
\end{tabular}

${ }^{a}$ All calculations performed at the gas-phase B3LYP 6-311G(d,p) geometry. Gas-phase excitation energies taken from the B3LYP TDDFT calculation. The solvent model is described in the text. ${ }^{b}$ Dipole moments and polarizabilites are taken from B3LYP (B3LYP/TDDFT) calculations for the ground state (excited states) in the 6-311G(d,p) basis. The cavity radius is taken as $4.32 \AA$. Dielectric constant and refractive indexes taken from ref 120 . The first entry includes the dispersion term, the second does not. ${ }^{c}$ Dipole moments and polarizabilites are taken from RHF (CIS) calculations for the ground state (excited state) in the 6-311G(d,p) basis. The cavity radius is taken as 4.32 A. Dielectric constant and refractive indexes taken from ref 120 . The first entry includes the dispersion term, the second does not. ${ }^{d}$ From Nad et al. ${ }^{119}$

(or $=$ nuclear, $\infty=$ electronic) and $y$ denoting the solute electronic state with which the solute reaction field is in equilibrium. In the calculation of the dispersion term (term containing $\Delta M$ ) we have used eq 3.23 from Kim, ${ }^{117}$ and in that case, we assume an isotropic average polarizability. In all other terms, we have not assumed that the polarizability is isotropic, and thus terms of the form $\mu_{\mathrm{S} 1} \Delta \alpha \mu_{\mathrm{S} 0}$ imply (row vector) matrix - (column vector) products. Results are presented with and without this dispersion term in Table 10. Because we have chosen a spherical geometry the reaction field factors can be treated as scalars.

In an Onsager model, the reaction field factors are defined by the size and shape of the solute cavity and the optical and static dielectric constants. The dielectric constants are obtained from Lange's handbook. ${ }^{120}$ The cavity radius is determined as follows. As noted in Table 2, the PCM model for solvation that uses a molecular shaped cavity in a dielectric continuum approach yields a ground state dipole moment for C151 in water that is approximately $1 \mathrm{D}$ larger than that of the Onsager model value with a cavity radius of $4.73 \AA$. We have thus determined the Onsager cavity radius using the ground state of $\mathrm{C} 151$, varying the radius from an Onsager dipole model in order to reproduce the solvated ground-state dipole moment obtained from a PCM treatment of C151 in water. In the Onsager calculations, we adjusted the cavity size using the B3LYP/6$311 \mathrm{G}(\mathrm{d}, \mathrm{p})$ dipole moment and polarizability tensor to represent C151 rather than an SCF procedure. This assumes a classical description of the solvent optical response, to yield results comparable with the Gaussian 98 solvation models. In this way, we obtained a cavity radius of $4.32 \AA$ (which was used for all solvents) for both ground and excited states in the calculations reported below. This radius yields a molecular volume slightly larger than that obtained from a volume calculation using G98. Although Kim has emphasized the importance of cavity size variations from solvent to solvent as well as with electronic state, ${ }^{116,117}$ the current limitations on accuracy of the gas-phase results only allow study of whether the DFT dipole moment differences yield semiquantitative agreement with experiment. Likewise, we have also used a spherical cavity rather than a oblate spheroidal cavity, as has been used to study C153 and related species. ${ }^{121}$ 
In Table 10, we present results for $\mathrm{C} 151$ with several solvent models based on (i) the gas-phase B3LYP or RHF 6-311G$(\mathrm{d}, \mathrm{p})$ ground-state dipole moments and polarizability tensors, (ii) the B3LYP/TDDFT gas-phase excitation energy to the $S_{1}$ state, and (iii) TDDFT or CIS dipole moments and polarizability tensor differences (computed using a finite field approach), using the model of Kim. ${ }^{116,117}$ Because we only compute the diagonal elements of the polarizability difference tensor, we assume that the off-diagonal elements of the excited state polarizability tensor are equal to those of the ground state in the calculations below. For the results including the dispersion term, the B3LYP/ TDDFT absorption energies are significantly lower than experimental excitation energies for the non-H-bonding solvents. The RHF/CIS values are also somewhat lower than the experimental values, but the error is reduced, relative to the B3LYP/TDDFT error. For the H-bonded solvents, both methods overestimate the excitation energies, suggesting significant specific solvent effects (H-bonding, see below). Neglect of the dispersion term yields much better agreement for the B3LYP/ TDDFT results for the non-H-bonding solvents, whereas the RHF/CIS error is considerably increased. Once again, both methods yield excitation energies that are too large for the H-bonding solvents.

The errors observed for solvated excitation energies stem from a number of sources. If the dispersion term is reasonably accurate, it suggests that the TDDFT dipole moment differences are somewhat large, leading to excessive stabilization of the excited state in solution. This would suggest that the CIS dipole moment difference is somewhat closer to the experimental value, which has been observed in calculations on C153. ${ }^{76,121}$ If the dispersion term is inaccurate, then the correct value for the dipole moment difference more likely lies between the TDDFT and CIS results. Another source of error stems from the fact that our calculations are not based on use of diffuse functions in the basis, and the results of Table 3 suggest this would lower our gas-phase excitation energy by about $0.1 \mathrm{eV}$. In addition, one might expect an overestimate of the gas-phase (FranckCondon) transition energy since in the reorganized modes we neglect the zero-point energy in the ground state in the calculation of the excitation energy. Finally, for H-bonding solvents, our calculations below indicate significant effects on the $S_{1}$ excitation energy for $\mathrm{H}$-bonding at the amine, and thus, the H-bonding solvents are expected to yield somewhat greater errors because the dielectric continuum treatment neglects specific solvation effects.

Nevertheless, the trends obtained with this simple model coupled with the DFT excitation energies and properties are quite good, and we expect that similar accuracy should be obtained for other coumarins.

D. Water-C151 Complexes. Topp et al. have recently published data that examine complexes of C151 and C153 with water and methanol in the gas phase.$^{67-70}$ Here, we focus on the single water-C151 complexes. The experimental results by Topp, et al. suggest the presence of two distinct water-C151 complexes. ${ }^{67,70}$ One has a $0-0 S_{1}$ excitation energy near that of $\mathrm{C} 151$, the second has an $\mathrm{S}_{1}$ excitation energy about $0.1 \mathrm{eV}$ lower than $\mathrm{C} 151$. The ionization potentials of the two complexes are markedly different (by approximately $0.5 \mathrm{eV}$ ). Topp et al. performed semiempirical geometry optimizations and found four local minima for a single water, one with a water $\mathrm{H}$-bonded at the carbonyl of $\mathrm{C} 151$, three others with a water $\mathrm{H}$-bonded at the amine (one with water donating an H-bond, the other two with a water accepting an H-bond). ${ }^{67,70}$ Their IR-UV double resonance spectroscopic results coupled with quantum chemical
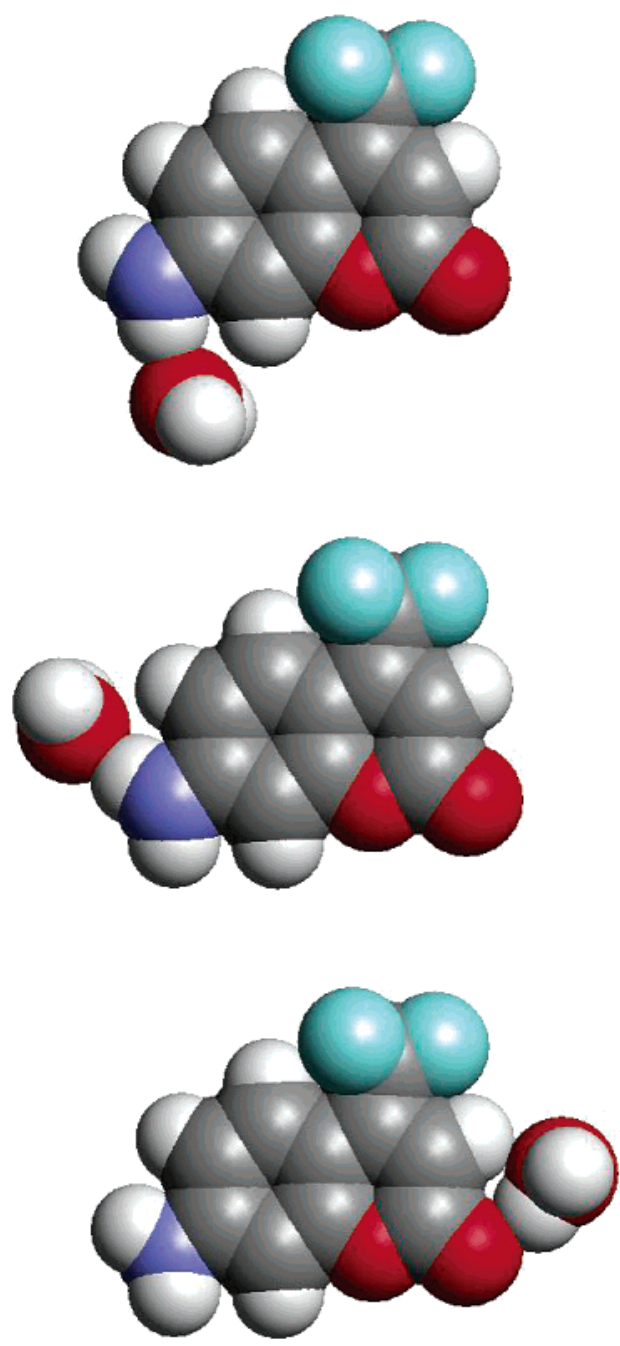

Figure 2. Geometries of the three $\mathrm{C} 151: \mathrm{H}_{2} \mathrm{O}$ dimers, as per Table 11 . Top: complex 1; center: complex 2; bottom: complex $\mathbf{3}$.

TABLE 11: Excitation Energies and Ionization Potentials for Various Water-C151 Complexes ${ }^{a}$

\begin{tabular}{ccccccc}
\hline complex $^{b}$ & $\begin{array}{c}\Delta E \\
(\mathrm{eV})^{c}\end{array}$ & $\begin{array}{c}\Delta S_{1} \\
(\mathrm{eV})\end{array}$ & $\begin{array}{c}\Delta S_{2} \\
(\mathrm{eV})\end{array}$ & $\begin{array}{c}\mathrm{IP}_{\text {vert }} \\
(\mathrm{eV})\end{array}$ & $\begin{array}{c}\mathrm{IP}_{\text {adiab }} \\
(\mathrm{eV})\end{array}$ & $\begin{array}{c}\mathrm{IP}_{\text {expt }} \\
(\mathrm{eV})^{d}\end{array}$ \\
\hline $\mathrm{C} 151$ & & 3.73 & 4.19 & 8.00 & 7.80 & 8.06 \\
1 & 0.0 & 3.60 & 4.17 & 7.63 & 7.37 & 7.66 \\
2 & 0.02 & 3.60 & 4.17 & 7.59 & 7.35 & 7.66 \\
3 & 0.01 & 3.70 & 4.22 & 8.08 & 7.72 & 8.17
\end{tabular}

${ }^{a}$ Methods discussed in section III D. ${ }^{b}$ Complexes identified in Figure 1. C151 is the uncomplexed Coumarin 151, presented for comparison. ${ }^{c}$ Energy difference between various water-C151 complexes, relative to the minimum energy structure. ${ }^{d}$ Experimental values Palmer et al. ${ }^{70}$ The assignments to specific structures are based on our theoretical results, as well as the results from Palmer et al. ${ }^{70}$

ground-state vibrational energy calculations indicate that the low IP/low excitation energy species involves the amine donating an H-bond to water, whereas the high IP/high excitation energy species involves water donating an $\mathrm{H}$-bond to the carbonyl. ${ }^{70}$ We performed a series of calculations on C151-water dimers to explore the spectroscopy of these species.

Figure 2 presents our three optimized geometries for C151: $\mathrm{H}_{2} \mathrm{O}$ complexes. Geometry coordinates are found in the Supporting Information. In Table 11 results are shown from three different local minima of the water-C151 complex (see Figure 1 and Supporting Information for geometries). In each case, we located an approximate energy minimum using AM1, and then performed a full geometry optimization using B3LYP with 
the $6-311 \mathrm{G}(\mathrm{d}, \mathrm{p})$ basis set. At each geometry, we then performed (a) a TDDFT/B3LYP calculation to obtain excitation energies, (b) a B3LYP calculation on the monocation of the complex to estimate a vertical ionization energy, and (c) a geometry optimization (B3LYP/6-311G(d,p)) of the monocation to estimate an adiabatic IP.

Of the three minima located for $\mathrm{C} 151: \mathrm{H}_{2} \mathrm{O}$ dimers, two correspond to water accepting an $\mathrm{H}$-bond from the amine, whereas the other corresponds to water $\mathrm{H}$-bond donation at the carbonyl oxygen. Though in general a carbonyl oxygen will have two H-bond accepting sites, a stable energy minimum was not found for the other $\mathrm{C} 151: \mathrm{H}_{2} \mathrm{O}$ during geometry optimization at the B3LYP/6-311G(d,p) level of theory. The relative energies of the structures are quite similar, with no more than a $0.5 \mathrm{kcal} /$ mol difference between the structures at the B3LYP/6-311G(d,p) level of theory. The two amine H-bonded structures yield $\mathrm{S}_{1}$ excitation energies lower than that of $\mathrm{C} 151$ by $0.13 \mathrm{eV}$, whereas the carbonyl structures yield $S_{1}$ excitations slightly lower $(0.03 \mathrm{eV})$ than $\mathrm{C} 151$. The two complexes have excitation energies differing by $0.1 \mathrm{eV}$, in agreement with Topp et al. Thus, our results suggest that the lower energy $S_{1}$ complex of Topp et al. corresponds to the amine-H-bonded complex. The IP calculations provide a consistency check for this assignment, in the sense that the lower $S_{1}$ state complex was also found to have a lower IP, and our amine $\mathrm{H}$-bonded complexes do indeed have lower IPs by 0.45 to $0.49 \mathrm{eV}$ than for the carbonyl H-bond dimers, whether one considers the vertical or adiabatic cases. Our assignments are thus in excellent agreement with the conclusions of Topp et al. based on the IR-UV double resonance studies. ${ }^{70}$

\section{Conclusions}

On the basis of the above results we are able to draw a number of interesting conclusions concerning C151 and C120. We find that TDDFT using hybrid functionals yields excitation energies in good agreement with experimental results (suitably corrected for solvent and/or internal reorganization effects). ZINDO S/CI excitation energies are found to be in good agreement with the all-electron methods used, but the dipole moment calculated for the $S_{1}$ state of $\mathrm{C} 120$ is significantly smaller than that based on other methods. We find similar ground state dipole moments for these two molecules, with the value of C120 slightly larger than that for C151. However, all of our estimates for the C151 and $\mathrm{C} 120$ ground state dipole moments are at least $0.5 \mathrm{D}$ larger than the experimental estimates. On the basis of the various methods used we suggest that the experimental estimates may be too low, or may represent a thermally averaged geometry in which rotation about the amine-ring bond leads to significantly lower ground-state dipole moments than for the equilibrium geometry. Our results indicate that the dipole moment difference between the ground and $\mathrm{S}_{1}$ state for $\mathrm{C} 151$ is approximately $3-4$ $\mathrm{D}$ in the gas-phase, and this is in good agreement with the estimates of Samanta et al., ${ }^{8}$ when corrected for solvation effects. Finally, we examined three water-C151 complexes, as studied by Topp et al. and are able to make an assignment of the two distinct complexes observed in their experiments. ${ }^{67,70}$ Overall, our results suggest that DFT and TDDFT will be useful and reasonably accurate tools for the exploration of the spectroscopy and properties of coumarins and related organic chromophores.

Acknowledgment. We wish to acknowledge financial support from the National Science Foundation (CHE-9731634 and CHE-9875091), and the donors of the Petroleum Research Fund. R.J.C. gratefully acknowledges a grant from the Rutgers University Nanochemistry Consortium.
Supporting Information Available: Expanded Tables 2-6. This material is available free of charge via the Internet at http:// pubs.acs.org.

\section{References and Notes}

(1) Dye Lasers, 3rd ed.; Schäfer, F. P., Ed.; Springer-Verlag: Berlin, New York, 1990; Vol. 1, p 244.

(2) Horng, M. L.; Gardecki, J. A.; Papazyan, A.; Maroncelli, M. J. Phys. Chem. 1995, 99, 17 311-37.

(3) Horng, M. L.; Gardecki, J. A.; Maroncelli, M. J. Phys. Chem. A 1997, 101, 1030-1047.

(4) Nemkovich, N. A.; Baumann, W.; Reis, H.; Zvinevich, Y. V. Proc. SPIE-Int. Soc. Opt. Eng. 1997, 2980, 390-399.

(5) Nemkovich, N. A.; Reis, H.; Baumann, W. J. Lumin. 1997, 71, $255-263$.

(6) Nemkovich, N. A.; Baumann, V.; Reis, H.; Zvinevich, Y. V.; Rubinov, A. N. Opt. Spektrosk. 1999, 87, 805-812.

(7) Parkanyi, C.; Antonious, M. S.; Aaron, J. J.; Buna, M.; Tine, A.; Cisse, L. Spectrosc. Lett. 1994, 27, 439-49. 8582 .

(8) Samanta, A.; Fessenden, R. W. J. Phys. Chem. A 2000, 104, 8577-

(9) Maroncelli, M.; Castner, E. W., Jr.; Webb, S. P.; Fleming, G. R. Springer Ser. Chem. Phys. 1986, 46, 303-7.

(10) Maroncelli, M.; Fleming, G. R. J. Chem. Phys. 1987, 86, 62216239.

(11) Maroncelli, M.; Fleming, G. R. J. Chem. Phys. 1988, 89, 875-81.

(12) Nagarajan, V.; Brearley, A. M.; Kang, T. J.; Barbara, P. F. J. Chem. Phys. 1987, 86, 3183-96.

(13) Kahlow, M. A.; Kang, T. J.; Barbara, P. F. J. Chem. Phys. 1988, $88,2372-8$

(14) Barbara, P. F.; Jarzeba, W. Adv. Photochem. 1990, 15, 1-68.

(15) Rosenthal, S. J.; Jimenez, R.; Fleming, G. R.; Kumar, P. V.; Maroncelli, M. J. Mol. Liquids 1994, 60, 25-56.

(16) Jimenez, R.; Fleming, G. R.; Kumar, P. V.; Maroncelli, M. Nature 1994, 369, 471-473.

(17) Vajda, S.; Jimenez, R.; Rosenthal, S. J.; Fidler, V.; Fleming, G. R.; Castner, E. W., Jr. J. Chem. Soc., Faraday Trans. 1995, 91, 867-873.

(18) Pal, H.; Nagasawa, Y.; Tominaga, K.; Kumazaki, S.; Yoshihara, K. J. Chem. Phys. 1995, 102, 7758-7760.

(19) Sarkar, N.; Datta, A.; Das, S.; Bhattacharyya, K. J. Phys. Chem. 1996, 100, 15483-15486.

(20) Sarkar, N.; Das, K.; Datta, A.; Das, S.; Bhattacharyya, K. J. Phys. Chem. 1996, 100, $10523-10527$.

(21) Riter, R. E.; Kimmel, J. R.; Undiks, E. P.; Levinger, N. E. J. Phys. Chem. B 1997, 101, 8292-8297.

(22) Riter, R. E.; Willard, D. M.; Levinger, N. E. J. Phys. Chem. B 1998, 102, 2705-2714

(23) Pant, D.; Levinger, N. E. Chem. Phys. Lett. 1998, 292, 200-206.

(24) Chudoba, C.; Nibbering, E. T. J.; Elsaesser, T. Dynamics of SiteSpecific Excited-State Solute-Solvent Interactions as Probed by Femtosecond Vibrational Spectroscopy. In Ultrafast Phenomena XI; Elsaesser, T., Fujimoto, J. G., Wiersma, D. A., Zinth, W., Eds.; Springer: Berlin, 1998; Vol. 63, pp 535-537.

(25) Nibbering, E. T. J.; Chudoba, C.; Elsaesser, T. Isr. J. Chem. 1999, $39,333-346$

(26) Nibbering, E. T. J.; Elsaesser, T. Appl. Phys. B: Lasers Opt. 2000 $71,439-441$

(27) Changenet-Barret, P.; Choma, C. T.; Gooding, E. F.; DeGrado, W. F.; Hochstrasser, R. M. J. Phys. Chem. B 2000, 104, 9322-9329.

(28) Frauchiger, L.; Shirota, H.; Uhrich, K. E.; Castner, E. W., Jr. J. Phys. Chem. B 2002, 106, 7463-7468.

(29) Shirota, H.; Castner, E. W., Jr. J. Chem. Phys. 2000, 112, 23672376.

(30) Jones, G. I.; Griffin, S. F.; Choi, C.-Y.; Bergmark, W. R. J. Org. Chem. 1984, 49, 2705-2708.

(31) Jones, G., II.; Jackson, W. R.; Choi, C. Y.; Bergmark, W. R. J Phys. Chem. 1985, 89, 294-300.

(32) Nagasawa, Y.; Yartsev, A. P.; Tominaga, K.; Johnson, A. E.; Yoshihara, K. J. Am. Chem. Soc. 1993, 115, 7922-7923.

(33) Nagasawa, Y.; Yartsev, A. P.; Tominaga, K.; Johnson, A. E.; Yoshihara, K. J. Chem. Phys. 1994, 101, 5717-5726.

(34) Nagasawa, Y.; Yartsev, A. P.; Tominaga, K.; Bisht, P. B.; Johnson, A. E.; Yoshihara, K. J. Phys. Chem. 1995, 99, 653-662.

(35) Yoshihara, K.; Yartsev, A.; Nagasawa, Y.; Kandori, H.; Douhal, A.; Kemnitz, K. Pure Appl. Chem. 1993, 65, 1671-1675.

(36) Yoshihara, K.; Nagasawa, Y.; Yartsev, A. P.; Kumazaki, S.; Kandori, H.; Johnson, A. E.; Tominaga, K. J. Photochem. Photobiol. A: Chem. 1994, 80.

(37) Yoshihara, K.; Tominaga, K.; Nagasawa, Y. Bull. Chem. Soc. Jpn. 1995, 68, 696-712 
(38) Yoshihara, K. Dynamical Aspects of Intermolecular Electron Transfer; International Symposium on Electron Transfer in Proteins and Supramolecular Assemblies at Interfaces, 1996, Shonan Village, Kanagawa, Japan.

(39) Yoshihara, K.; Pal, H.; Shirota, H.; Nagasawa, Y.; Tominaga, K. Effects of Vibrations in Chemical Dynamics: High-Frequency Vibrational and Low-Frequency Solvent Dynamical Effects on Intermolecular Electron Transfer. In Los Alamos National Laboratory Report, Presented at the Seventh International Conference on Time-Resolved Vibrational Spectroscopy, Los Angeles, California, 1995; LA-13290-C, 69-73.

(40) Shirota, H.; Pal, H.; Tominaga, K.; Yoshihara, K. Chem. Phys. 1998 $236,355-364$.

(41) Shirota, H.; Pal, H.; Tominaga, K.; Yoshihara, K. J. Phys. Chem. A 1998, 102, 3089-3102.

(42) Pal, H.; Shirota, H.; Tominaga, K.; Yoshihara, K. J. Chem. Phys. 1999, 110, $11454-11465$.

(43) Yoshihara, K. Ultrafast Intermolecular Electron Transfer in Solution. In Electron Transfer: From Isolated Molecules to Biomolecules, Part 2; Adv. Chem. Phys. 1999, 107, 371-402.

(44) Castner, E. W., Jr.; Kennedy, D.; Cave, R. J. J. Phys. Chem. A 2000, 103, 2869-2885.

(45) Zerner, M. C.; Loew, G. H.; Kirchner, R. F.; Mueller-Westerhoff U. T. J. Am. Chem. Soc. 1980, 102, 589

(46) Cave, R. J.; Newton, M. D. Chem. Phys. Lett. 1996, 249, 15-19.

(47) Cave, R. J.; Newton, M. D. J. Chem. Phys. 1997, 106, 9213.

(48) Koch, W.; Holthausen, M. C. A Chemist's Guide to Density Functional Theory, 2nd ed.; Wiley-VCH: Weinheim, 2001.

(49) Parr, R. G.; Yang, W. Density Functional Theory of Atoms and Molecules; Oxford: New York, 1989.

(50) Petersilka, M.; Gossman, U. J.; Gross, E. K. U. Phys. Rev. Lett. 1996, 76, 1212

(51) Casida, M. E. Time-Dependent Density Functional Response Theory for Molecules. In Recent Advances in Density Functional Theory Methods, Part I; Chong, D. P., Ed.; World Scientific: Singapore, 1995; pp 155193.

(52) Casida, M. E. Time-Dependent Density Functional Response Theory of Molecular Systems: Theory, Computational Methods, and Functionals In Recent Developments and Applications of Modern Density Functional Theory; Seminario, J. M., Ed.; Elsevier: Amsterdam, 1996; p 391.

(53) Runge, E.; Gross, E. K. U. Phys. Rev. Lett. 1984, 52, 997.

(54) Adamo, C.; Barone, V. J. Chem. Phys. 1998, 108, 664-675.

(55) Adamo, C.; Barone, V. Chem. Phys. Lett. 1999, 314, 152-157.

(56) Adamo, C.; Scuseria, G. E.; Barone, V. J. Chem. Phys. 1999, 111, 2889-2899

(57) Adamo, C.; Barone, V. Chem. Phys. Lett. 2000, 330, 152-160. 464

(58) Bauernschmitt, R.; Ahlrichs, R. Chem. Phys. Lett. 1996, 256, 454-

(59) Bauernschmitt, R.; Ahlrichs, R.; Hennrich, F. H.; Kappes, M. M. J. Am. Chem. Soc. 1998, 120, 5052-5059.

(60) Beck, M. E.; Rebentisch, R.; Hohlneicher, G.; Fulscher, M. P.; Serrano-Andres, L.; Roos, B. O. J. Chem. Phys. 1997, 107, 9464-9474.

(61) Hirata, S.; Head-Gordon, M.; Bartlett, R. J. J. Chem. Phys. 1999, 111, $10774-10786$.

(62) Hirata, S.; Head-Gordon, M. Chem. Phys. Lett. 1999, 314, 291299

(63) Hirata, S.; Lee, T. J.; Head-Gordon, M. J. Chem. Phys. 1999, 111, 8904-8912.

(64) van Gisbergen, S. J. A.; Rosa, A.; Ricciardi, G.; Baerends, E. J. J. Chem. Phys. 1999, 111, 2499-2506.

(65) Guillaumont, D.; Nakamura, S. Dyes Pigm. 2000, 46, 85-92.

(66) Jean, J. M.; Hall, K. B. Proc. Natl. Acad. Sci. U.S.A. 2001, 98, 37-41.

(67) Pryor, B. A.; Palmer, P. M.; Andrews, P. M.; Berger, M. B.; Topp,

M. R. J. Phys. Chem. A 1998, 102, 3284-3292.

(68) Pryor, B. A.; Palmer, P. M.; Chen, Y.; Topp, M. R. Chem. Phys. Lett. 1999, 299, 536-544.

(69) Palmer, P. M.; Chen, Y.; Topp, M. R. Chem. Phys. Lett. 2000 321, 62-70.

(70) Palmer, P. M.; Chen, Y.; Topp, M. R. Chem. Phys. Lett. 2000 318, 440-447.

(71) Redchenko, V. V.; Safronov, A. I.; Kirpichenok, M. A.; Grandberg, I. I.; Traven, V. F. Zh. Obshch. Khim. 1992, 62, 2313-18.

(72) McCarthy, P. K.; Blanchard, G. J. J. Phys. Chem. 1993, 97, 12 20512209

(73) Raineri, F. O.; Perng, B.-C.; Friedman, H. L. Electrochim. Acta 1997, 42, 2749-2761

(74) Muhlpfordt, A.; Schanz, R.; Ernsting, N. P.; Farztdinov, V.; Grimme, S. Phys. Chem. Chem. Phys. 1999, 1, 3209-3218. 6484.
(76) Matyushov, D. V.; Newton, M. D. J. Phys. Chem. A 2001, 105, $8516-8532$

(77) Morimoito, A.; Yatsuhashi, T.; Shimada, T.; Biczok, L.; Tryk, D. A.; Inoue, H. J. Phys. Chem. A 2001, 105, $10488-10496$.

(78) Finley, J.; Malmqvist, P. A.; Roos, B. O.; Serrano-Andres, L. Chem. Phys. Lett. 1998, 288, 299-306.

(79) Roos, B. O.; Andersson, K.; Fulscher, M. P.; Malmqvist, P. A.; Serrano-Andres, L.; Pierloot, K.; Merchan, M. Multiconfigurational perturbation theory: Applications in electronic spectroscopy. Adv. Chem. Phys. 1996, 93, 137-144

(80) Roos, B. O. Acc. Chem. Res. 1999, 32, 137-144. 724

(82) Hariharan, P. C.; Pople, J. A. Mol. Phys. 1974, 27, 209. 2257.

(84) Clark, T.; Chandrasekhar, J.; Sptiznagel, G. W.; Schleyer, P. v. R. J. Comput. Chem. 1983, 4, 294.

(85) Krishnan, R.; Binkley, J. S.; Seeger, R.; Pople, J. A. J. Chem. Phys. 1980, 72,650 .

(86) McLean, A. D.; Chandler, G. S. J. Chem. Phys. 1980, 72, 5639.

(87) Sadlej, A. J. Collect. Czech. Chem. Commun. 1988, 53, 1995.

(88) Becke, A. D. J. Chem. Phys. 1993, 98, 5648.

(89) Dewar, M. J. S.; Zoebisch, E. G.; Healy, E. F. J. Am. Chem. Soc. 1985, 107, 3902-3909.

(90) Stratmann, R. E.; Scuseria, G. E.; Frisch, M. J. J. Chem. Phys. 1998, 109, 8218-8224.

(91) Becke, A. D. Phys. Rev. A 1988, 38, 3098.

(92) Lee, C.; Yang, W.; Parr, R. G. Phys. Rev. B 1988, 37, 785. 3865 .

93) Perdew, J. P.; Burke, K.; Ernzerhof, M. Phys. Rev. Lett. 1996, 77,

(94) Perdew, J. P.; Burke, K.; Ernzerhof, M. Phys. Rev. Lett. 1997, 78, 1396.

(95) Perdew, J. P.; Ernserhof, M.; Burke, K. J. Chem. Phys. 1996, 105, 9982.

(96) Adamo, C.; Barone, V. J. Chem. Phys. 1999, 110, 6158-6170.

(97) Kurth, S.; Perdew, J. P.; Blaha, P. Int. J. Quantum Chem. 1999. 75,889 .

(98) Cave, R. J.; Castner, E. W., Jr. Submitted to J. Phys. Chem. A, 2002.

(99) Champagne, B.; Perpete, E. A.; Gisbergen, S. J. A. v.; Baerends E. J.; Snijders, J. G.; Soubra-Ghaoui, C.; Robins, K. A.; Kirtman, B. J. Chem. Phys. 1998, 109, $10489-10498$.

(100) Miertus, S.; Scrocco, E.; Tomasi, J. Chem. Phys. 1981, 55, 117

(101) Miertus, S.; Tomasi, J. Chem. Phys. 1982, 65, 239.

(102) Cossi, M.; Barone, V.; Cammi, R.; Tomasi, J. Chem. Phys. Lett 1996, $255,327$.

(103) Wong, M. W.; Frisch, M. J.; Wiberg, K. B. J. Am. Chem. Soc 1991, 113, 4776-4782.

(104) Cossi, M.; Barone, V. J. Phys. Chem. A 2000, 104, 10 614-10 622

(105) Cossi, M.; Barone, V. J. Chem. Phys. 2000, 112, 2427-2435.

(106) Cossi, M.; Rega, N.; Scalmani, G.; Barone, V. J. Chem. Phys. 2001, 114, 5691-5701.

(107) Cossi, M.; Barone, V. J. Chem. Phys. 2001, 115, 4708-4717.

(108) Frisch, M. J.; Trucks, G. W.; Schlegel, H. B.; Scuseria, G. E.; Robb, M. A.; Cheeseman, J. R.; Zakrzewski, V. G.; Montgomery, J. A.; Jr.; Stratmann, R. E.; Burant, J. C.; Dapprich, S.; Millam, J. M.; Daniels, A. D.; Kudin, K. N.; Strain, M. C.; Farkas, O.; Tomasi, J.; Barone, V. Cossi, M.; R. Cammi; Mennucci, B.; Pomelli, C.; Adamo, C.; Clifford, S.; Ochterski, J.; Petersson, G. A.; Ayala, P. Y.; Cui, Q.; Morokuma, K.; Salvador, P.; Dannenberg, J. J.; Malick, D. K.; Rabuck, A. D.; Raghavachari, K.; Foresman, J. B.; Cioslowski, J.; Ortiz, J. V.; Baboul, A. G.; Stefanov, B. B.; Liu, G.; Liashenko, A.; Piskorz, P.; Komaromi, I.; Gomperts, R.; Martin, R. L.; Fox, D. J.; Keith, T.; Al-Laham, M. A.; Peng, C. Y.; Nanayakkara, A.; Challacombe, M.; Gill, P. M. W.; Johnson, B.; Chen, W.; Wong, M. W.; Andres, J. L.; Gonzalez, C.; Head-Gordon, M.; Replogle, E. S.; Pople, J. A. Gaussian 98, revision A.11.3 ed.; Gaussian, Inc.: Pittsburgh, PA, 2001

(109) Andersson, K.; Barysz, M.; Bernhardsson, A.; Blomberg, M. R. A.; Carissan, Y.; Cooper, D. L.; Cossi, M.; Fleig, T.; Fülscher, M. P.; Gagliardi, L.; Graaf, C. d.; Hess, B. A.; Karlström, G.; Lindh, R.; Malmqvist, P.-A.; Neogrády, P.; Olsen, J.; Roos, B. O.; Schimmelpfennig, B.; Schütz, M.; Seijo, L.; Serrano-Andrés, L.; Siegbahn, P. E. M.; Stålring, J.; Thorsteinsson, T.; Veryazov, V.; Wierzbowska, M.; Widmark, P.-O. Molcas Version 5.2; Lund University: Sweden, 2001.

(110) Zerner, M. C. Zindo electronic structure theory code, 3.7 ed.; Molecular Simulations, Inc.: La Jolla, CA, 1991.

(111) Burke, K.; Ernzerhof, M.; Perdew, J. P. Chem. Phys. Lett. 1997, $265,115-120$.

(112) Ernzerhof, M.; Scuseria, G. E. J. Chem. Phys. 1999, 110, 5029

(113) Moylan, C. R. J. Phys. Chem. 1994, 98, 13513-16.

(114) Garzillo, C.; Improta, R.; Peluso, A. Theochem 1998, 426, 145153 
(115) Novak, I.; Kovac, B. J. Electron Spectrosc. Relat. Phenom. 2000, $113,9-13$.

(116) Kim, H. J. J. Chem. Phys. 1996, 105, 6818-6832.

(117) Kim, H. J. J. Chem. Phys. 1996, 105, 6833-6843.

(118) Parusel, A. B. J.; Rettig, W.; Sudholt, W. J. Phys. Chem. A 2002, $106,804-815$.

(119) Nad, S.; Pal, H. J. Phys. Chem. A 2001, 105, 1097-1106.
(120) Lange. Lange's Handbook of Chemistry; McGraw-Hill: New York, 1973.

(121) Chowdhury, A.; Locknar, S. A.; Premvardhan, L. L.; Peteanu, L. A. J. Phys. Chem. A 1999, 103, 9614-9625.

(122) Ravi, M.: Soujanya, T.; Samanta, A.; Radhakrishnan, T. P. $J$. Chem. Soc., Faraday Trans. 1995, 91, 2739-42.

(123) Rechthaler, K.; Koehler, G. Chem. Phys. 1994, 189, 99-116. 